\title{
Is advanced life support better than basic life support in prehospital care? A systematic review
}

\author{
Olli-Pekka Ryynänen ${ }^{1,2}$, Timo lirola ${ }^{3}$, Janne Reitala ${ }^{4}$, Heikki Pälve ${ }^{5}$, Antti Malmivaara ${ }^{6 *}$
}

\begin{abstract}
Background -: Prehospital care is classified into ALS- (advanced life support) and BLS- (basic life support) levels according to the methods used. ALS-level prehospital care uses invasive methods, such as intravenous fluids, medications and intubation. However, the effectiveness of ALS care compared to BLS has been questionable.

Aim -: The aim of this systematic review is to compare the effectiveness of ALS- and BLS-level prehospital care.

Material and methods -: In a systematic review, articles where ALS-level prehospital care was compared to BLSlevel or any other treatment were included. The outcome variables were mortality or patient's health-related quality of life or patient's capacity to perform daily activities.

Results -: We identified 46 articles, mostly retrospective observational studies. The results on the effectiveness of ALS in unselected patient cohorts are contradictory. In cardiac arrest, early cardiopulmonary resuscitation and defibrillation are essential for survival, but prehospital ALS interventions have not improved survival. Prehospital thrombolytic treatment reduces mortality in patients having a myocardial infarction. The majority of research into trauma favours BLS in the case of penetrating trauma and also in cases of short distance to a hospital. In patients with severe head injuries, ALS provided by paramedics and intubation without anaesthesia can even be harmful. If the prehospital care is provided by an experienced physician and by a HEMS organisation (Helicopter Emergency Medical Service), ALS interventions may be beneficial for patients with multiple injuries and severe brain injuries. However, the results are contradictory.

Conclusions -: ALS seems to improve survival in patients with myocardial infarction and BLS seems to be the proper level of care for patients with penetrating injuries. Some studies indicate a beneficial effect of ALS among patients with blunt head injuries or multiple injuries. There is also some evidence in favour of ALS among patients with epileptic seizures as well as those with a respiratory distress.
\end{abstract}

\section{Introduction}

Prehospital care is an essential part of the treatment process in many acute diseases and trauma. Prehospital care is usually classified into ALS- (advanced life support) and BLS-(basic life support) treatment levels according to the methods used [1]. ALS refers to sophisticated prehospital care using invasive methods, such as intravenous fluids, medications and intubation. The vehicle used in ALS has either been a ground ambulance (GA) or a helicopter. Basic Life Support (BLS) is medical care which is used to assure patient's vital functions until the patient has been transported to

\footnotetext{
* Correspondence: antti.malmivaara@thl.fi

${ }^{6}$ Centre for Health and Social Economics, Insitute for Health and Welfare,

Mannerheimintie 166, 00270 Helsinki, Finland

Full list of author information is available at the end of the article
}

appropriate medical care. ALS-level prehospital care has usually been implemented by physicians or paramedics, while BLS-level care is given by paramedics or emergency medical technicians. However, in most cases ALS units use the same techniques as BLS units.

While the concepts associated with ALS and BLS are diverse and differ between countries, both have developed towards greater sophistication. Some procedures that were previously classified as ALS-level prehospital care are now also available as part of BLS.

In spite of active research, the effectiveness of ALS care compared to BLS has been questioned [2]. Several research reports have been published, though no final conclusion has been drawn. Research projects have used different methods and target groups, and results have been controversial. The implementation of prehospital 
care is strongly dependent on local political, geographical, cultural and economic factors, making comparisons between systems difficult. The effectiveness of prehospital care also depends on the transportation method used and the emergency care given in the hospital. Thus, the problem of the effectiveness of ALS compared to BLS is only one link in the whole emergency care chain.

In emergency care, two alternative strategies have generally been presented [3]:

1. scoop and run: the patient is transported to a high level hospital as quickly as possible, with minimal prehospital treatments

2. stay and play: the patient is stabilized on site before transportation.

While debate on the merits of these two strategies is still ongoing, their division has been criticized for oversimplifying the problems of emergency care. Moreover, the two strategies do not correspond exactly to the division between ALS and BLS prehospital treatments. In the United States, the scoop and run strategy has been favoured, whereas in Europe several emergency systems use a stay and play -approach.

Researching and comparing studies in emergency care is difficult. Two main problems arise: Finding a suitable comparator across individual studies and also difficulties in comparing studies performed within different health care systems. ALS and BLS also entail different protocols in different countries.

Emergency care is affected by several elements:

- amount of population in an operational area

- geographical variables such as lakes, rivers, mountains

- quality and network of roads

- location and level of hospitals

- distribution of accident risk in the operational area

- amount, distribution, dispatching and quality of emergency units

- education of the personnel

- alarm systems

- communication technology, e.g. mobile phones, telemedicine

- development of the traffic: quality of vehicles and roads, traffic jams

The need for ALS procedures is quite rare and mostly ALS and BLS units provide the same levels of care. The factors influencing emergency care are not constant; they may change rapidly. The whole treatment chain can be totally different at night compared to the daytime.

The aim of this systematic review is to compare the effectiveness of ALS and BLS. The review covers all patient groups (e.g. trauma, cardiac disease, cardiac arrest, respiratory distress, convulsions) and all vehicles used for transportation of the team/patient (GA, helicopter, or both). The full report has been published in Finnish (available from: http://finohta.stakes.fi/EN/index. htm) [4].

\section{Methods}

\section{Data Sources}

The literature search was conducted from the following databases: PubMed, preMEDLINE OVID Medline, CRD databases, Cochrane database of systematic reviews, EBM reviews, CINAHL. To explore the grey literature, we made a search from the Internet by using Google Scholar search engine. The review period covered the years 1995-2008. All languages were included.

Combinations of the following search terms were used: advanced life support, basic life support, ALS (not amyotroph*), BLS, emergency medical services, emergency treatment, advanced cardiac life support, emergency, trauma, thrombolytic therapy, thromboly* fibrinoly*, prehospital, pre-hospital out-of-hospital (care or treatment or management or triage), paramedic, technician, ambulance*, helicopter, HEMS, mobile unit.

We performed a related articles -search from the PubMed for all articles we included after reading the abstracts. We also checked the reference lists from all relevant articles. The search process is presented in Figure 1.

We also prepared a general overview of the previous reviews on the effectiveness of prehospital care and helicopter emergency services. The search strategy was the same as that used in the literature search for the systematic review.

\section{Selection criteria}

Articles were included if they fulfilled at least one of the following criteria:

1. ALS prehospital care was compared to the BLS, or 2. two different ALS systems were compared (e.g. physician-ALS compared to paramedic-ALS), or 3. ALS prehospital care was compared to any other treatment (e.g. ALS care compared to patient transport by laypersons).

4. A comparison between ALS and BLS was done virtually by an expert group.

The accepted outcome variables were:

1. Survival with a follow-up period until discharge from the hospital or later, or

2. Patient's health-related quality of life or capacity to perform daily activities at follow-up. 


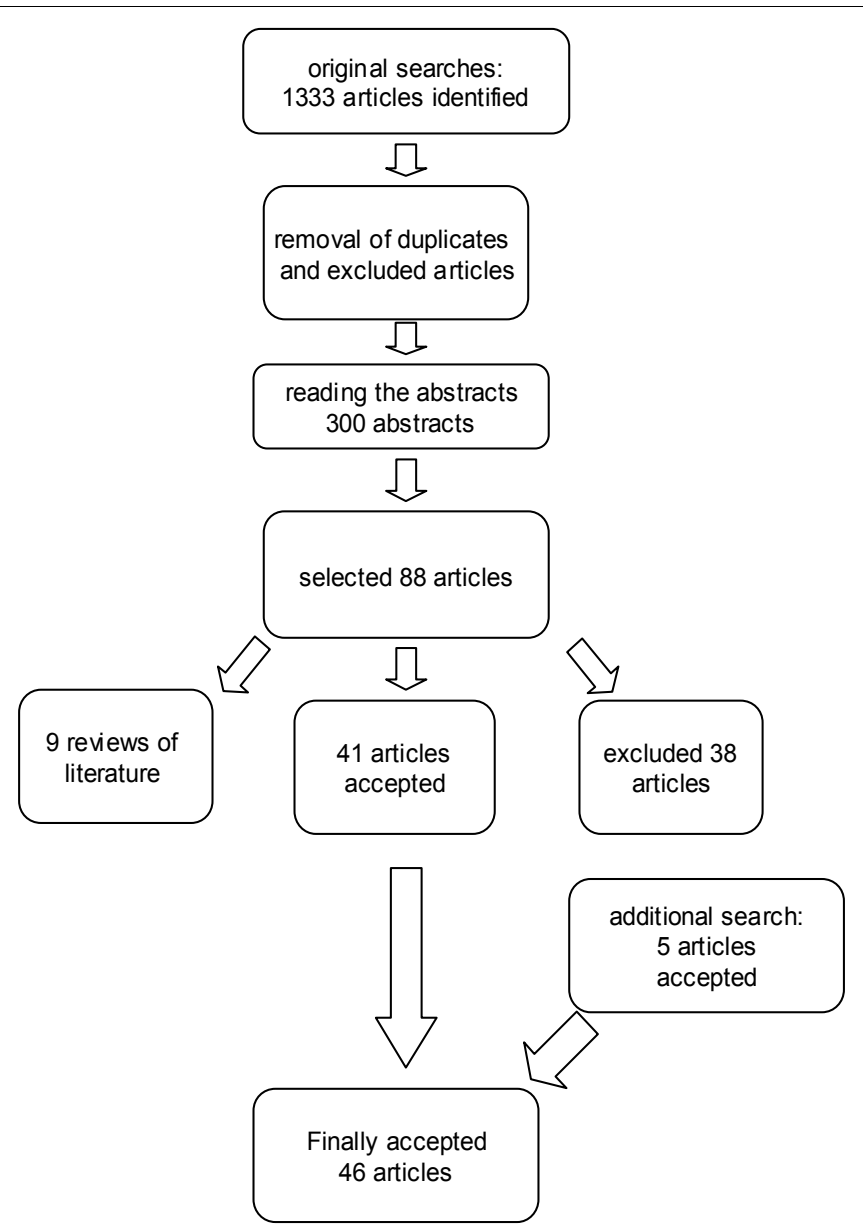

Figure 1 Flow diagram of the search process

This systematic review is focused on patients' secondary survival. We accepted only the studies with a followup period until hospital discharge or later. We considered that studies using survival until arrival to the hospital are sensitive to the transport system and distance. We did not accept articles that only discussed treatment practices or treatment delays. Also we excluded articles using surrogate outcomes such as blood pressure or pain. Articles based on geographical epidemiology were also excluded.

Studies concerning thrombolytic therapy were included if the thrombolysis was given by a prehospital emergency care unit (e.g. in an ambulance). Thus, studies in which the thrombolysis was performed by a general practitioner were excluded.

\section{Interventions}

BLS was defined as a prehospital emergency service using non-invasive life-saving procedures including cardiopulmonary resuscitation, bleeding control, splinting broken bones, artificial ventilation, basic airway management and administration of oral or rectal medications.
Use of a semi-automatic defibrillator was considered to be a part of BLS. Some BLS systems are allowed to use adrenaline in resuscitation. They were accepted as BLS if they were referred as a BLS in the article. BLS is usually provided by emergency medical technicians (EMT) or other similarly trained professionals.

ALS was defined as a prehospital emergency medical service using invasive life-saving procedures including all procedures of BLS but including advanced airway management, intravenous infusions and medications, synchronized cardioversion, cardiac monitoring, electrocardiogram interpretation and other procedures conventionally used at the hospital level. ALS is provided by physicians, paramedics or by other specially trained professionals.

\section{Data extraction}

The following data were gathered from all the included articles: Bibliographical data (author, title, journal, year, volume, issue, pages), research aim, research methods (prospective, retrospective), years of gathering data, 
place of research (state or other), description of the research population, including patients' age and severity of disease (expressed as injury severity scale or other), professionals involved (physician, paramedic, EMT), transportation method, transportation time and distance, available and used treatments, treatment delays, baseline differences in the research population, mortality, healthrelated quality of life or capacity to perform daily activities, adjusted outcomes, transferability of research population and treatments across jurisdictions, amount of drop-outs and blinded measurement of outcome variables.

The articles were classified as follows:

1. Randomized controlled trials.

2. Studies where ALS care was compared to cases where ALS care was requested but not obtained.

3. Prospective studies using the TRISS-methodology or another comparable method to adjust the comparison between ALS and BLS or other control.

4. Epidemiological studies with a follow-up of all emergency patients and comparison between ALS and BLS.

5. Quasi-experimental studies with a comparison of ALS in one area with BLS in another area.

6. Before and after -studies using ALS and BLS data gathered in the same area at a different time.

7. Retrospective case-control studies using matched controls.

8. Studies based on expert panels.

The fulfilment of search criteria and the validity and methodological quality of all included studies were assessed by two independent researchers. In cases of controversy, a third researcher was consulted until a consensus was reached. Besides classification based on study design the following quality assessments of each individual study were made: unselected patients recruited, baseline differences, number of drop-outs described and blinded outcome assessment.

\section{Results}

We found 1333 references from the databases. In additional searches, five articles were identified. Two researchers read the abstracts independently as well as identified the original articles. Altogether, 46 articles were included. Additionally, we identified eight previous meta-analyses or reviews [1,5-11]. The search process is presented in Figure 1. A summary of previous reviews is presented in Table 1 and original articles in Table 2 [12-57].

Of the 46 studies there was one randomized controlled trial [13], 15 prospective follow-up studies $[19,20,24,30,32,37-39,42,45,47,50,53,55,57]$ while the rest had a retrospective design. In the randomised trial [13] the effectiveness of prehospital thrombolysis and inhospital thrombolysis for acute myocardial infarction was compared. Only two of the prospective studies reported an acceptable follow-up of patients [38,47].

\section{Studies concerning all patient groups}

Five of the included studies [23,31,34,36,45] made no distinction between surgical, internal or other patients. None of those articles were considered to be of high quality. The reported result was the same in three articles $[23,36,45]$ : No difference between ALS and BLS was found. Two articles $[31,34]$ used a specialist group to assess the effectiveness of ALS without comparison to BLS.

\section{Prehospital thrombolysis for myocardial infarction}

Two studies concerning thrombolytic treatment of cardiac infarction were published in 1995 [12,13] with three further articles on the topic published some ten years later $[40,46,49]$. In the two studies of 1995 , patients having thrombolytic treatment showed a trend to better survival than patients having thrombolysis given in a hospital, but the result was not statistically significant.

In the three studies from 2004-2005, prehospital thrombolytic treatment was more effective than hospital thrombolysis, but only in Björklund's study [49] the result was statistically significant. The validity and generalisability of the studies were considered good.

\section{Cardiac arrest}

The role of ALS in cardiac arrest was studied in nine studies $[17,18,20,26,28,42,47,54,55]$. In one study [54] ALS care showed better survival rates than BLS. In five studies patients treated by ALS and in one study patients treated by BLS showed a trend to better survival than patients in the control group, but the results were not statistically significant. One study was concentrated in traumatic cardiac arrest, with no difference between ALS and BLS [47]

\section{Penetrating and unselected traumas}

We found eleven articles that dealt with penetrating or unselected trauma $[16,19,21,22,25,27,39,43,50,56,57]$. One included study [56] focused exclusively on penetrating trauma. Eight included studies considered both penetrating and blunt traumas, two of which $[43,57]$ presented results separately for each type of injury.

No difference between ALS and BLS was found in five of these studies $[19,21,25,50,57]$, though injuries in those studies were relatively mild (ISS over 15 in $11-15 \%$ of patients). Five of the studies showed better results for BLS $[16,22,27,39,56]$. In studies by Seamon 
Table 1 Summary of findings in the previous reviews on effectiveness of advanced vs. basic life support.

\begin{tabular}{|c|c|c|c|c|c|}
\hline Reference & $\begin{array}{l}\text { Author(s) } \\
\text { of review, } \\
\text { year, } \\
\text { country }\end{array}$ & description of a review & $\begin{array}{l}\text { contents of the } \\
\text { review }\end{array}$ & assessment of the review & conclusion \\
\hline 5 & $\begin{array}{l}\text { Liberman } \\
\text { et al. 2000, } \\
\text { Canada }\end{array}$ & $\begin{array}{l}\text { non-systematic review, } \\
\text { traumas only }\end{array}$ & $\begin{array}{l}15 \text { studies from } \\
\text { years 1983-1997; } \\
\text { classification } \\
\text { according to quality: } \\
\text { 1. medium quality } 5 \\
\text { studies favouring } \\
\text { ALS, } 1 \text { study } \\
\text { favouring BLS. } \\
\text { 2.high quality: } 1 \\
\text { favouring ALS, } 1 \\
\text { study favouring BLS } \\
\text { 3. very high quality: } \\
1 \text { favouring ALS, } 6 \\
\text { favouring BLS }\end{array}$ & $\begin{array}{l}\text { In general the quality of studies } \\
\text { was poor, many studies quite old, } \\
\text { the follow-up periods starting even } \\
\text { from 1930's. }\end{array}$ & $\begin{array}{l}7 \text { studies favourintg ALS, } \\
8 \text { studies favouring BLS. } \\
\text { Studies of higher quality favouring } \\
\text { BLS. }\end{array}$ \\
\hline 6 & $\begin{array}{l}\text { Sethi et al. } \\
2000 \\
\text { England }\end{array}$ & $\begin{array}{l}\text { A systematic Cochrane- } \\
\text { review }\end{array}$ & & Only one study included & No difference between ALS and BLS \\
\hline 7 & $\begin{array}{l}\text { Nicholl et } \\
\text { al. 2003, } \\
\text { England }\end{array}$ & $\begin{array}{l}\text { A systematic review on } \\
\text { the effectiveness of } \\
\text { helicopter services }\end{array}$ & 36 original studies & $\begin{array}{l}\text { HEMS better than ground } \\
\text { transportation, mortality } \mathrm{OR}=0,86 \text {, } \\
\text { not statistically significant. }\end{array}$ & \\
\hline 8 & $\begin{array}{l}\text { Koskinen } \\
2005, \\
\text { Finland }\end{array}$ & $\begin{array}{l}\text { thesis for master's degree } \\
\text { in health economics, } \\
\text { contains a non-systematic } \\
\text { review }\end{array}$ & 36 original studies & $\begin{array}{l}\text { In general the quality of studies } \\
\text { was poor }\end{array}$ & $\begin{array}{l}\text { cost-effectiveness of a helicopter } \\
\text { service was assessed to be } 5750 \\
€ / \text { life year gained (confidence } \\
\text { interval } 2000-24500 € \text { ) }\end{array}$ \\
\hline 9 & $\begin{array}{l}\text { Isenberg } \\
\text { and Bissel, } \\
2005, \\
\text { Canada }\end{array}$ & $\begin{array}{l}\text { A non-systematic review, } \\
\text { four parts: } \\
\text { 1. trauma } \\
\text { 2. cardiac arrest } \\
\text { 3. cardiac infarct } \\
\text { 4. distubances of } \\
\text { consciousness }\end{array}$ & $\begin{array}{l}20 \text { original articles, } 2 \\
\text { meta-analyses from } \\
\text { years 1984-2004 }\end{array}$ & $\begin{array}{l}\text { 1. Trauma: } 14 \text { studies, } 8 \text { favouring } \\
\text { ALS, } 6 \text { BLS. All new studies } \\
\text { favouring BLS } \\
\text { 2. cardiac arrest: early resuscitation } \\
\text { and defibrillation associated with } \\
\text { better survival, no special effect of } \\
\text { ALS detected. } \\
\text { 3. Cardiac infarct: } 1 \text { study, no } \\
\text { difference between ALS and BLS. } \\
\text { 4. Disturbances of consciousness, } 1 \\
\text { study, no difference between ALS } \\
\text { and BLS. }\end{array}$ & $\begin{array}{l}\text { In general results favouring BLS. } \\
\text { Review for paramedic-ALS only, } \\
\text { physician-ALS excluded. }\end{array}$ \\
\hline 10,11 & $\begin{array}{l}\text { Thomas } \\
2004, \\
\text { Thomas } \\
2007\end{array}$ & $\begin{array}{l}\text { Qualitative review, } \\
\text { renovation by a new } \\
\text { version }\end{array}$ & & & No conclusion \\
\hline 1 & $\begin{array}{l}\text { Liberman } \\
2007\end{array}$ & $\begin{array}{l}\text { Opinion-based article } \\
\text { about trauma treatment, } \\
\text { grounded by a non- } \\
\text { systematic review }\end{array}$ & & & In general favouring BLS. \\
\hline
\end{tabular}

[56] and Demetriades et al. [16], ALS treatment of trauma was compared with transportation to hospital by laymen. In both studies, transportation by a layperson showed better survival rates than ALS.

Three studies [22,27,39] showed better survival in BLS patients as well as the study by Stiell et al [57] among subgroup of patients with GCS $<9$. In the study by Frankema et al. [43] blunt trauma patients having ALS treatment given by a physician and transported by a helicopter showed better survival than BLS-treated patients transported by a GA.

In general, there is no evidence that ALS would be superior compared to BLS in penetrating or unselected traumas. There is one study supporting ALS by
Frankema et al. [43] but the result was confounded by the transportation method.

\section{Blunt head injury}

Six studies concentrated in blunt head injury $[24,29,30,38,44,48]$. In three studies the combination of ALS treatment and helicopter transportation gave better results than BLS with a GA $[24,29,48]$. In two studies $[38,44]$ intubation without medication by a paramedic was harmful compared to intubation in a hospital by a physician using medication to assist intubation. In the study by Di Bartolomeo [30], there was no difference between ALS and BLS. 
Table 2 Summary of findings in the articles presenting effectiveness of advanced vs. basic life support.

\begin{tabular}{|c|c|c|c|c|c|}
\hline $\begin{array}{l}\text { Reference } \\
\text { no }\end{array}$ & $\begin{array}{l}\text { research, } \\
\text { author, } \\
\text { country, } \\
\text { publication } \\
\text { year. }\end{array}$ & $\begin{array}{l}\text { type on the study } \\
\text { illness or injury. } \\
\text { research population. } \\
n(A L S), n(B L S) \text {. } \\
\text { severity of disease or injury }\end{array}$ & $\begin{array}{l}\text { the implementer of the } \\
\text { care. } \\
\text { ALS, BLS. } \\
\text { transport. } \\
\text { ALS, BLS. } \\
\text { treatments. }\end{array}$ & $\begin{array}{l}\text { Outcome, mortality, } \\
\text { other outcome } \\
\text { variables, results }\end{array}$ & conclusion \\
\hline 12 & $\begin{array}{l}\text { Shuster et al. } \\
1995 \\
\text { Canada }\end{array}$ & $\begin{array}{l}\text { prospective chart review. } \\
\text { acute cardiac disease. } \\
\text { ALS } n=1821 \\
\text { BLS } n=1245\end{array}$ & $\begin{array}{l}\text { ALS-PARAMEDIC, GA. } \\
\text { BLS-EMT, GA. } \\
\text { an urban setting with } \\
\text { short transportation } \\
\text { times (less than } 10 \\
\text { minutes) }\end{array}$ & $\begin{array}{l}\text { mortality ALS } 16,5 \% \\
\text { BLS } 19,5 \% \\
\text { risk of death } \\
\text { ALS OR }=1 \\
\text { BLS OR } 1,12(0,78-1,61)\end{array}$ & $\begin{array}{l}\text { no difference between the } \\
\text { groups }\end{array}$ \\
\hline 13 & $\begin{array}{l}\text { Boissel } 1995 \\
\text { France }\end{array}$ & $\begin{array}{l}\text { multicentre study in } 16 \\
\text { countries, PHT compared with } \\
\text { thrombolysis in a hospital. } \\
\text { ALS (immediate PTH) } n=2750 \text {. } \\
\text { BLS (hospital throbolysis) } n= \\
2719 \text {. }\end{array}$ & $\begin{array}{l}\text { ALS-MD, GA. } \\
\text { BLS-MD, GA. } \\
\text { both groups treated by } \\
\text { a physician. }\end{array}$ & $\begin{array}{l}\text { 30-day mortality ALS } \\
9,7 \%, \text { BLS } 11,1 \% \\
\text { adjusted } p=0,08\end{array}$ & trend to favour PHT (ALS). \\
\hline 14 & $\begin{array}{l}\text { Alldredge et } \\
\text { al., California, } \\
\text { U.S.A., } \\
1995\end{array}$ & $\begin{array}{l}\text { retrospective chart review. } \\
\text { children with status epilepticus, } \\
\text { ALS } n=19 \text { (treatment on site) } \\
\text { BLS } n=26 \text { (treatment in a } \\
\text { hospital) }\end{array}$ & $\begin{array}{l}\text { ALS-PARAMEDIC, GA } \\
\text { BLS-EMT, GA } \\
\text { prehospital diazepam } \\
\text { therapy (given rectally } \\
\text { or intravenously) }\end{array}$ & $\begin{array}{l}\text { duration of status } \\
\text { epilepticus ALS } 32 \text { min, } \\
\text { BLS } 60 \text { min ( } p=0,007) \\
\text { repeated cramps ALS } \\
56 \%, \text { BLS } 85 \% \text { ( } p= \\
0,045) \text {, mortality } 0 \%\end{array}$ & favours ALS. \\
\hline 15 & $\begin{array}{l}\text { Adams et } \\
\text { al.1996 } \\
\text { Illinois, U.S.A. }\end{array}$ & $\begin{array}{l}\text { retrospective study. } \\
\text { declined level of (epilepsy, } \\
\text { hypoglycaemia, stroke). } \\
\text { ALS } n=113, \text { BLS } n=90\end{array}$ & $\begin{array}{l}\text { ALS-paramedic, GA } \\
\text { BLS-EMT, GA }\end{array}$ & $\begin{array}{l}\text { mortality ALS } 6 \%, n=7 \\
\text { BLS } 2 \%, n=2\end{array}$ & $\begin{array}{l}\text { no difference between the } \\
\text { groups }\end{array}$ \\
\hline 16 & $\begin{array}{l}\text { Demetriades et } \\
\text { al. } 1996 \\
\text { California, U.S. } \\
\text { A. }\end{array}$ & $\begin{array}{l}\text { retrospective, all traumas } \\
\text { ALS or } B L S n=4856 \\
\text { private transport } n=926\end{array}$ & $\begin{array}{l}\text { ALS-PARAMEDIC ori } \\
\text { BLS-EMT compared } \\
\text { with patients } \\
\text { transported by a private } \\
\text { vehicle }\end{array}$ & $\begin{array}{l}\text { mortality: ALS or BLS } \\
9,3 \% \\
\text { private transport } 4,0 \% \\
\text { adjusted RR } 1.60(\mathrm{P}= \\
.002) .\end{array}$ & $\begin{array}{l}\text { better survival and less } \\
\text { permanently disabled in } \\
\text { privately transported patients }\end{array}$ \\
\hline 17 & $\begin{array}{l}\text { Silfvast and } \\
\text { Ekstrand } \\
1996 \\
\text { Finland }\end{array}$ & $\begin{array}{l}\text { before-after-design, prehospital } \\
\text { cardiac arrest before (Period I, } \\
\text { retrospective) and after (Period } \\
\text { II, prospective) reorganisation of } \\
\text { the EMS system. } \\
\text { Phase I: ALS-PHYSICIAN } \\
\text { experienced physicians, } \mathrm{n}= \\
\text { 444 } \\
\text { Phase II: ALS-PHYSICIAN junior } \\
\text { physicians, } \mathrm{n}=395\end{array}$ & $\begin{array}{l}\text { two ALS-systems. } \\
\text { physicians experienced } \\
\text { (Phase I) and less } \\
\text { experienced (Phase II), } \\
\text { both operated with a } \\
\text { GA }\end{array}$ & $\begin{array}{l}\text { total mortality: } \\
\text { Phase I } 90.8 \% \\
\text { Phase II } 91,6 \% \text { (NS) } \\
\text { survival of patients with } \\
\text { ventricular fibrillation } \\
\text { phase I: } 41(34 \%) \\
\text { phase II: } 33(25 \%) p= \\
0,05\end{array}$ & $\begin{array}{l}\text { no difference between groups } \\
\text { in total mortality. } \\
\text { among patients with ventricular } \\
\text { fibrillation better results in phase I }\end{array}$ \\
\hline 18 & $\begin{array}{l}\text { Nguyen-Van- } \\
\text { Tam et al. } \\
1997 \\
\text { England }\end{array}$ & $\begin{array}{l}\text { retrospective cohort } \\
\text { cardiac arrest } \\
\text { ALS } n=285 \\
\text { BLS } n=144 \\
\text { dual response } n=79\end{array}$ & $\begin{array}{l}\text { ALS-PARAMEDIC, BLS- } \\
\text { EMT } \\
\text { dual response: both ALs } \\
\text { and BLS in the scene. } \\
\text { GA in all groups. }\end{array}$ & $\begin{array}{l}\text { mortality: ALS 91,9\%, } \\
\text { dual } 98,7 \%, \text { BLS 93,8\%, p } \\
=0,63) \\
\text { ALS adjusted survival RR } \\
1,21(0,50-2,91)\end{array}$ & $\begin{array}{l}\text { no difference between ALS, BLS } \\
\text { and dual response groups }\end{array}$ \\
\hline 19 & $\begin{array}{l}\text { Rainer et al. } \\
\text { 1997a } \\
\text { England }\end{array}$ & $\begin{array}{l}\text { prospective } \\
\text { trauma patients } \\
\text { ALS } n=247 \\
\text { BLS } n=843\end{array}$ & $\begin{array}{l}\text { ALS-PARAMEDIC, ALS- } \\
\text { EMT } \\
\text { ALS GA } \\
\text { BLS GA } \\
\end{array}$ & $\begin{array}{l}\text { mortality: ALS 4\%, BLS } \\
\text { 3\% (NS) } \\
\text { TRISS: unexpected } \\
\text { deaths: } \\
\text { ALS } n=5, \text { BLS } n=18 \\
\text { unexpected survivals: } \\
\text { ALS } n=6, \text { BLS } n=9 \text {, } \\
\text { (NS) }\end{array}$ & $\begin{array}{l}\text { no difference between ALS and } \\
\text { BLS groups }\end{array}$ \\
\hline 20 & $\begin{array}{l}\text { Rainer et al. } \\
\text { 1997b England }\end{array}$ & $\begin{array}{l}\text { prospective } \\
\text { cardiac arrest } \\
\text { ALS } n=111 \\
\text { BLS } n=110\end{array}$ & $\begin{array}{l}\text { ALS-PARAMEDIC, BLS- } \\
\text { EMT } \\
\text { ALS GA } \\
\text { BLS GA }\end{array}$ & $\begin{array}{l}\text { mortality ALS 93\%, BLS } \\
94 \% \\
\text { p = 0,59. resuscitation by } \\
\text { a bystander and early } \\
\text { defibrillation associated } \\
\text { with better survival }\end{array}$ & $\begin{array}{l}\text { no difference between ALS and } \\
\text { BLS groups }\end{array}$ \\
\hline 21 & $\begin{array}{l}\text { Suominen et } \\
\text { al. } 1998 \\
\text { Finland }\end{array}$ & $\begin{array}{l}\text { retrospective } \\
\text { pediatric trauma, ALS } n=49 \\
\text { BLS } n=72 \text {, total material } \\
n=288\end{array}$ & $\begin{array}{l}\text { ALS-PHYSICIAN, BLS- } \\
\text { EMT } \\
\text { ALS helicopter and GA, } \\
\text { BLS GA }\end{array}$ & $\begin{array}{l}\text { ALS } 22,4 \% \\
\text { BLS } 31,9 \%(N S)\end{array}$ & $\begin{array}{l}\text { no difference between groups, a } \\
\text { subgroup ISS } 25-49 \text { ALS better } \\
(p=0,04)\end{array}$ \\
\hline
\end{tabular}


Table 2 Summary of findings in the articles presenting effectiveness of advanced vs. basic life support. (Continued)

\begin{tabular}{|c|c|c|c|c|c|}
\hline 22 & $\begin{array}{l}\text { Nicholl et al. } \\
1998 \\
\text { Sheffield, } \\
\text { England }\end{array}$ & $\begin{array}{l}\text { retrospective } \\
\text { trauma } \\
\text { ALS } n=882 \\
\text { BLS } n=331\end{array}$ & $\begin{array}{l}\text { ALS-PARAMEDIC, BLS- } \\
\text { EMT } \\
\text { GA in both groups }\end{array}$ & $\begin{array}{l}6 \text { months follow-up: } \\
\text { mortality } \\
\text { ALS 6,0\%, BLS 4,6\% } \\
\text { OR 2,02 (1,05-3,89) } \\
\text { ALS: higher mortality in } \\
\text { penetrating trauma and } \\
\text { large fractures }\end{array}$ & higher mortality in ALS \\
\hline 23 & $\begin{array}{l}\text { Eisen and } \\
\text { Dubinsky } \\
\text { 1998, Canada }\end{array}$ & $\begin{array}{l}\text { retrospective } \\
\text { all patient groups in } \\
\text { prehospital care } \\
\text { BLS } n=1000 \\
\text { ALS } n=397\end{array}$ & $\begin{array}{l}\text { ALS-PARAMEDIC (level } \\
2 \text { and level 3, level } 1= \\
\text { BLS), BLS-EMT GA in } \\
\text { both }\end{array}$ & $\begin{array}{l}\text { mortality: ALS 5,8\%, BLS } \\
4,6 \% \text { (NS), LOS. no } \\
\text { difference between } \\
\text { groups }\end{array}$ & no difference between groups \\
\hline 24 & $\begin{array}{l}\text { Abbott et al. } \\
1998 \\
\text { California U.S.A. }\end{array}$ & $\begin{array}{l}\text { prospective case-control } \\
\text { closed head injury } \\
\text { ALS-PHYSICIAN n = } 196 \\
\text { ALS-PARAMEDIC n = } 1090 \\
\text { HEMS manned by nurse or } \\
\text { nurse/paramedic/physician }\end{array}$ & $\begin{array}{l}\text { ALS-HEMS } \\
\text { ALS-PARAMEDIC } \\
\text { ALS-PHYSICIAN } \\
\text { helicopter } \\
\text { ALS-PARAMEDIC GA }\end{array}$ & $\begin{array}{l}\text { ALS-PHYSICIAN 20\% } \\
\text { ALS-PARAMEDIC 31\% } \\
\text { OR 1,75 } \\
1,21-2,53 \\
\text { subgroups: age, GCS had } \\
\text { effect on mortality }\end{array}$ & $\begin{array}{l}\text { ALS-HEMS better than ALS- } \\
\text { PARAMEDIC }\end{array}$ \\
\hline 25 & $\begin{array}{l}\text { Owen et al. } \\
1999 \\
\text { Texas, U.S.A. }\end{array}$ & $\begin{array}{l}\text { retrospective TRISS } \\
\text { trauma patients, comparison } \\
\text { between helicopter and GA, } \\
\text { ALS-PARAMEDIC (GA) n = } 687 \\
\text { ALS-PARAMEDIC (helicopter) n } \\
=105\end{array}$ & $\begin{array}{l}\text { ALS-PARAMEDIC (GA) } \\
\text { ALS-PARAMEDIC, ALS-N } \\
\text { (helicopter) } \\
\text { ALS-PARAMEDIC (GA) } \\
\text { ALS-PARAMEDIC, ALS-N } \\
\text { (helicopter) }\end{array}$ & $\begin{array}{l}\text { mortality: 14,3\%, 6,0\% } \\
\text { TRISS: GA predicted } 39 \\
\text { deaths, actually 41, } \\
\text { helicopter: predicted } 16 \\
\text { deaths, actually } 15\end{array}$ & no difference between groups \\
\hline 26 & $\begin{array}{l}\text { Mitchell et al. } \\
2000 \text { Scotlandi }\end{array}$ & $\begin{array}{l}\text { before-after design } \\
\text { cardiac arrest, } \\
\text { period } 1 \mathrm{n}=259 \\
\text { period } 2 \mathrm{n}=294\end{array}$ & ALS-PARAMEDIC, GA & $\begin{array}{ll}\text { period } 1 & 94,2 \% \\
\text { period } 2 & 93,5 \%\end{array}$ & no difference between groups \\
\hline 27 & $\begin{array}{l}\text { Eckstein et al. } \\
2000 \\
\text { California, U.S. } \\
\text { A. }\end{array}$ & $\begin{array}{l}\text { retrospective } \\
\text { serious trauma } \\
\text { ALS } n=93 \\
\text { BLS } n=403\end{array}$ & $\begin{array}{l}\text { ALS-PARAMEDIC, BLS- } \\
\text { EMT, } \\
\text { ALS GA, BLS GA }\end{array}$ & $\begin{array}{l}\text { mortality ALS 93\%, BLS } \\
67 \% \\
\text { adjusted 5,3 (2,3-14,2) }\end{array}$ & higher mortality in ALS \\
\hline 28 & $\begin{array}{l}\text { Pitetti et al. } \\
2001 \\
\text { Pennsylvania, } \\
\text { U.S.A. }\end{array}$ & $\begin{array}{l}\text { retrospective } \\
\text { pediatric cardiac arrest } \\
\text { ALS-PARAMEDIC } n=150 \\
\text { BLS-EMT } n=39\end{array}$ & $\begin{array}{l}\text { ALS-PARAMEDIC } \\
\text { BLS-EMT } \\
\text { ALS GA, BLS GA }\end{array}$ & $\begin{array}{l}\text { ALS } 96,7 \% \\
\text { BLS } 0 \%(N S)\end{array}$ & $\begin{array}{l}\text { no difference between ALS and } \\
\text { BLS groups }\end{array}$ \\
\hline 29 & $\begin{array}{l}\text { Garner et al. } \\
2001 \\
\text { Australia }\end{array}$ & $\begin{array}{l}\text { retrospective comparison } \\
\text { between two ALS-systems } \\
\text { blunt trauma in head } \\
\text { ALS-PARAMEDIC } n=250 \\
\text { ALS-PHYSICIAN } n=46\end{array}$ & $\begin{array}{l}\text { comparison of two } \\
\text { levels of ALS } \\
\text { ALS-PARAMEDIC GA } \\
\text { ALS-PHYSICIAN } \\
\text { helicopter }(91 \%)\end{array}$ & $\begin{array}{l}\text { mortality: ALS-PHYSICIAN } \\
20 \% \\
\text { ALS-PARAMEDIC } 31 \% \\
\text { survival ALS-PARAMEDIC } \\
\text { OR }=1, \text { ALS-PHYSICIAN } \\
\text { OR }=2,70(1,48-4,95)\end{array}$ & $\begin{array}{l}\text { ALS-PHYSICIAN better than ALS- } \\
\text { PARAMEDIC }\end{array}$ \\
\hline 30 & $\begin{array}{l}\text { Di Bartolomeo } \\
\text { et al. } 2001 \\
\text { Italy }\end{array}$ & $\begin{array}{l}\text { ALS patients compared with } \\
\text { cases when ALS was requested } \\
\text { but not obtained } \\
\text { Serious brain injury } \\
\text { ALS-PHYSICIAN } \mathrm{n}=92 \\
\text { BLS-H } \mathrm{n}=92\end{array}$ & $\begin{array}{l}\text { ALS-PHYSICIAN } \\
\text { helicopter } \\
\text { BLS-H GA }\end{array}$ & $\begin{array}{l}\text { mortality: ALS 30\%, BLS } \\
24 \% \\
\text { adjusted no difference }\end{array}$ & no difference between groups \\
\hline 31 & $\begin{array}{l}\text { Kurola et al. } \\
2002 \\
\text { Finland }\end{array}$ & $\begin{array}{l}\text { expert panel } \\
\text { all prehospital patients, } \\
\text { specialist appraisal, ALS- } \\
\text { PHYSICIAN } n=206\end{array}$ & $\begin{array}{l}\text { ALS-PHYSICIAN } \\
\text { helicopter and GA }\end{array}$ & $\begin{array}{l}\text { mortality } 10,6 \% \text {, no } \\
\text { compatison, specialist } \\
\text { appraisal }\end{array}$ & $\begin{array}{l}1,5 \% \text { of patients benefit of ALS- } \\
\text { treatment, } 20.4 \% \text { partial benefit }\end{array}$ \\
\hline 32 & $\begin{array}{l}\text { Bjerre et al. } \\
2002 \\
\text { Danmark }\end{array}$ & $\begin{array}{l}\text { chronic pulmonary disease } \\
\text { ALS } n=67, B L S n=72\end{array}$ & $\begin{array}{l}\text { ALS-PHYSICIAN, BLS- } \\
\text { EMT } \\
\text { ALS GA, BLS GA }\end{array}$ & $\begin{array}{l}\text { mortality: ALS 15\%, BLS } \\
24 \%\end{array}$ & $\begin{array}{l}\text { ALS-PHYSICIAN better survival } \\
\text { than BLS-EMT }\end{array}$ \\
\hline 33 & $\begin{array}{l}\text { Thomas et al. } \\
2002 \\
\text { Massachusetts, } \\
\text { U.S.A. }\end{array}$ & $\begin{array}{l}\text { retrospective } \\
\text { blunt trauma, ALS-PARAMEDIC } \\
\text { helicopter } n=2292 \\
\text { ALS-PARAMEDIC GA, } n=3245 \text {, } \\
\text { BLS-EMT GA } n=7723\end{array}$ & $\begin{array}{l}3 \text { groups: ALS- } \\
\text { PARAMEDIC GA, ALS- } \\
\text { PHYSICIAN helicopter, } \\
\text { BLS-EMT GA }\end{array}$ & $\begin{array}{l}\text { mortality: } 9,4 \% \text { (ALS } \\
\text { helicopter or GA), BLS } \\
\text { 3,0\%; helicopter vs GA: } \\
\text { OR 0,756 }(0,59-0,98), \text { BLS } \\
\text { vs ALS 0,42 }(0,32-0,56)\end{array}$ & $\begin{array}{l}\text { higher mortality in ALS than BLS } \\
\text { higher mortality in GA than } \\
\text { helicopter }\end{array}$ \\
\hline 34 & $\begin{array}{l}\text { Lossius et al. } \\
\text { Norway } 2002\end{array}$ & $\begin{array}{l}\text { expert panel } \\
\text { all prehospital patients, ALS } n= \\
1062\end{array}$ & $\begin{array}{l}\text { ALS-PHYSICIAN } \\
40 \% \text { helicopter } \\
\text { transport, } 60 \% \text { GA }\end{array}$ & $\begin{array}{l}\text { mortality } 20,7 \% \text {, specialist } \\
\text { appraisal } 7 \%(n=74) \\
\text { benefit fromALS-care }\end{array}$ & ALS useful, no controls \\
\hline
\end{tabular}


Table 2 Summary of findings in the articles presenting effectiveness of advanced vs. basic life support. (Continued)

\begin{tabular}{|c|c|c|c|c|c|}
\hline 35 & $\begin{array}{l}\text { Lee et al. } 2002 \\
\text { Australia }\end{array}$ & $\begin{array}{l}\text { retrospective } \\
\text { blunt trauma, head injury } \\
\text { ALS-PARAMEDIC } n=1167 \\
\text { ALS-PHYSICIAN } n=224 \\
\text { BLS level } 3 n=452 \\
\text { BLS level } 4 n=45 \\
\text { BLS other } n=96\end{array}$ & $\begin{array}{l}\text { ALS-PHYSICIAN } \\
\text { ALS-PARAMEDIC } \\
\text { BLS-EMT (2 different } \\
\text { levels) } \\
\text { ALS GA, BLS GA }\end{array}$ & $\begin{array}{l}\text { mortality: } \\
\text { ALS-PARAMEDIC 24,8\%, } \\
\text { ALS-PHYSICIAN 19,6\% } \\
\text { BLS level } 312,2 \%, \text { BLS } \\
\text { level } 4 \text { 13,3\%, BLS other } \\
21 \% \\
\text { Adjusted: BLS OR = } 1 \\
\text { ALS-PHYSICIAN OR = } \\
4,27 \\
\text { ALS-PARAMEDIC OR = } \\
\text { 2,18 }\end{array}$ & $\begin{array}{l}\text { higher mortality in ALS } \\
\text { higer mortality in ALS- } \\
\text { PHYSICIAN than in ALS- } \\
\text { PARAMEDIC }\end{array}$ \\
\hline 36 & $\begin{array}{l}\text { Cristenzen et } \\
\text { al. } 2003 \\
\text { Danmark }\end{array}$ & $\begin{array}{l}\text { retrospective before-after } \\
\text {-design } \\
\text { all prehospital patients } \\
\text { ALS-PHYSICIAN } n=795+35 \\
\text { BLS-EMT } n=4989 \text {. } \\
\text { before-after -study: in the } \\
\text { second phase } 28 \% \text { of cases } \\
\text { treated by ALS }\end{array}$ & $\begin{array}{l}\text { ALS-PHYSICIAN } \\
\text { BLS-EMT } \\
\text { ALS GA } \\
\text { BLS GA }\end{array}$ & $\begin{array}{l}\text { phase I mortality } 10,0 \% \\
\text { mortality in phase } \|= \\
10,5 \% \\
\text { phase } \| \text { mortality in ALS- } \\
\text { group } 14,7 \% \text {, phase II } \\
\text { mortality in BLS-group } \\
8,9 \%(p<0,001) \\
\text { OR } 1,06 \text { (NS) }\end{array}$ & $\begin{array}{l}\text { total mortality same in both } \\
\text { periods }\end{array}$ \\
\hline 37 & $\begin{array}{l}\text { Osterwalder } \\
2003 \\
\text { Switzerland }\end{array}$ & $\begin{array}{l}\text { prospective TRISS } \\
\text { multiple trauma } \\
\text { ALS } n=196 \\
\text { BLS } n=71\end{array}$ & $\begin{array}{l}\text { ALS-PHYSICIAN, } \\
\text { BLS-P, BLS-EMT } \\
\text { EMT lower level } \\
\text { education } \\
\text { ALS GA or helicopter, } \\
\text { BLS GA }\end{array}$ & $\begin{array}{l}\text { mortality in ALS } 11,2 \% \\
\text { BLS } 14,1 \% \text { (NS) } \\
\text { predicted mortality in } \\
\text { ALS } 23,3 \% \text {, actual } \\
\text { mortality } 22 \% \\
\text { predicted mortality in } \\
\text { BLS } 6,6 \% \\
\text { actual mortality } 10 \%\end{array}$ & $\begin{array}{l}\text { ALS trend to lower mortality } \\
\text { than BLS }\end{array}$ \\
\hline 38 & $\begin{array}{l}\text { Bochiccio et al. } \\
2003 \\
\text { Maryland, U.S. } \\
\text { A. }\end{array}$ & $\begin{array}{l}\text { prospective retrospective } \\
\text { brain injury: } \\
\text { blunt ( } 92 \%) \text {, penetrating (8\%), } \\
\text { comparison between patients } \\
\text { intubated on site and those } \\
\text { intubated in hospital } \\
\text { intubated on site } n=78 \\
\text { intubated in hospital } n=113\end{array}$ & $\begin{array}{l}\text { all ALS-PARAMEDIC } \\
67 \% \text { had helicopter } \\
\text { transport, others with } \\
\text { GA }\end{array}$ & $\begin{array}{l}\text { mortality: intubated on } \\
\text { site } 23 \% \\
\text { intubated in hospital } \\
12,4 \%(p=0,005)\end{array}$ & $\begin{array}{l}\text { higher mortality in patients } \\
\text { intubated on site }\end{array}$ \\
\hline 39 & $\begin{array}{l}\text { Liberman et al. } \\
\text { Canada, } 2003\end{array}$ & $\begin{array}{l}\text { prospective epidemiological } \\
\text { study } \\
\text { all traumas } \\
\text { Montreal ALS } n=801 \\
\text { Montreal BLS } n=4295 \\
\text { Toronto ALS } n=1000 \\
\text { Toronto BLS } n=1530 \\
\text { Quebec BLS } n=1779\end{array}$ & $\begin{array}{l}\text { Montreal ALS- } \\
\text { PHYSICIAN } \\
\text { Montreal BLS-EMT } \\
\text { Toronto ALS- } \\
\text { PARAMEDIC } \\
\text { Toronto EMT-BLS } \\
\text { Quebec BLS-EMT } \\
\text { ALS GA, BLS GA }\end{array}$ & $\begin{array}{l}\text { ALS } 29 \% \\
\text { ISS } 25-4930 \% \\
\text { ISS } 50-7679 \% \\
\text { BLS } 18 \% \\
\text { ISS } 25-4926 \% \\
\text { ISS } 50-7676 \% \\
\text { ALS-PHYSICIAN vs BLS } \\
1,36^{*} \\
\text { ALS-PARAMEDIC vS BLS } \\
1,06^{* *} \text {, ALS-PHYSICIAN vS } \\
\text { ALS-PARAMEDIC 1,20** } \\
\text { ALS VS BLS 1,21** * } P= \\
0,01 \\
*{ }^{*} p=\text { NS }\end{array}$ & higher mortality in ALS \\
\hline 40 & $\begin{array}{l}\text { Danchin et al. } \\
2004 \\
\text { France }\end{array}$ & $\begin{array}{l}\text { retrospective chart review } \\
\text { PHT } n=180 \\
\text { hospital trombolysis } n=365 \\
\text { CABG, } P C l \\
n=434 \\
\text { no reperfusion } n=943\end{array}$ & $\begin{array}{l}96 \% \text { of PHT-patients } \\
\text { got treatment } \\
\text { from"mobile intensive } \\
\text { care unit" } \\
\text { all transported by GA }\end{array}$ & $\begin{array}{l}\text { PHT } 6 \% \text { (1 year mortality) } \\
\text { hospital thrombolysis } \\
11 \% \\
\text { PCI } 11 \% \text {, no reperfusion } \\
\text { treatment } 21 \% \text {, PHT } \\
\text { mortality } \\
\text { RR } 0,49(0,24-1,00)\end{array}$ & $\begin{array}{l}\text { lowest mortality in } \mathrm{PHT} \\
\text { Comparison between } \mathrm{PHT} \text { and } \\
\text { other reperfusion treatment } \\
\mathrm{RR}=0,52(\mathrm{p}=0,08)\end{array}$ \\
\hline 41 & $\begin{array}{l}\text { Biewener et al. } \\
2004 \\
\text { Germany }\end{array}$ & $\begin{array}{l}\text { prospective TRISS } \\
\text { multiple trauma } n=403,4 \\
\text { groups } \\
\text { 1) HEMS-UNI } n=140 \\
\text { 2) AMB-REG } n=102 \\
\text { 3) AMB-UNI } n=70 \\
\text { 4) INTER } n=91\end{array}$ & $\begin{array}{l}\text { all four goups ALS- } \\
\text { PHYSICIAN } \\
\text { 1) university hospital } \\
\text { 2)regional hospital } \\
\text { 3) university hospital } \\
\text { 4) local hospital } \\
\text { 1)transport by } \\
\text { helicopter } \\
\text { 2-4) transport by a GA }\end{array}$ & $\begin{array}{l}\text { mortality rates: } \\
\text { 1) } 22,1 \% \\
\text { 2) } 41,2 \% \\
\text { 3) } 15,7 \% \\
\text { 4) } 17,6 \% \\
\text { adusted risk } \\
\text { 1) } \mathrm{OR}=1 \\
\text { 2) } \mathrm{OR}=1,06 \mathrm{NS} \\
\text { 3) } \mathrm{OR}=4,06, \mathrm{P}<0,05 \\
\text { 4) } \mathrm{OR}=1,28, \mathrm{NS}\end{array}$ & $\begin{array}{l}\text { ALS-PHYSICIAN + helicopter } \\
\text { transport to university hospital is } \\
\text { better than transport by a GA to } \\
\text { regional hospital } \\
\text { no difference in mortality } \\
\text { between HEMS-UNI and AMB- } \\
\text { UNI }\end{array}$ \\
\hline
\end{tabular}


Table 2 Summary of findings in the articles presenting effectiveness of advanced vs. basic life support. (Continued)

\begin{tabular}{|c|c|c|c|c|c|}
\hline 42 & $\begin{array}{l}\text { Stiell et al. } \\
2004 \\
\text { Canada }\end{array}$ & $\begin{array}{l}\text { before-after -design } \\
\text { cardiac arrest } \\
\text { ALS } n=4247 \\
\text { BLS } n=1391\end{array}$ & $\begin{array}{l}\text { ALS-PARAMEDIC } \\
\text { BLS-EMT } \\
\text { ALS GA, BLS GA }\end{array}$ & $\begin{array}{l}\text { mortality } \\
\text { ALS 95,0\% } \\
\text { BLS } 94,9 \%(p=0,83) \\
\text { no adjustment } \\
\text { No difference in QoL or } \\
\text { cerebral performance }\end{array}$ & No difference in mortality. \\
\hline 43 & $\begin{array}{l}\text { Frankema et al. } \\
2004 \\
\text { Netherlands }\end{array}$ & $\begin{array}{l}\text { retrospective } \\
\text { all serious injuries } \\
\text { ALS } n=107 \\
\text { BLS } n=239\end{array}$ & $\begin{array}{l}\text { ALS-PHYSICIAN } \\
\text { BLS-EMT, ALS } \\
\text { helicopter, BLS GA }\end{array}$ & $\begin{array}{l}\text { mortality: ALS } 34,5 \%, \text { BLS } \\
24,3 \% \\
\text { adjustment: patients } \\
\text { treated by ALS } 2,4 \text { fold } \\
\text { probability to survive ( } p \\
=0,076 \text { ). } \\
\text { Blunt trauma OR 2,8, p = } \\
0,036 \text {, penetrating } \\
\text { trauma } 0,2 \text { (NS) }\end{array}$ & ALS better survival \\
\hline 44 & $\begin{array}{l}\text { Wang et al. } \\
2004 \\
\text { Pennsylvania, } \\
\text { U.S.A. }\end{array}$ & $\begin{array}{l}\text { retrospective epidemiological } \\
\text { study } \\
\text { brain injury, comparison } \\
\text { between patients intubated } \\
\text { prehospitally with patients } \\
\text { intubated in the hospital } \\
\text { intubation on-site } n=1797 \\
\text { intubated in a hospital } n= \\
2301\end{array}$ & $\begin{array}{l}\text { on-site intubation by } \\
\text { ALS-PARAMEDIC or by } \\
\text { ALS-PHYSICIAN, } \\
\text { transportation by } \\
\text { helicopter or by a GA }\end{array}$ & $\begin{array}{l}\text { mortality on-site } \\
\text { intubaltion } 48,5 \% \text {, } \\
\text { hospitla intubation } \\
28,2 \% \text {, adjusted OR 3,99 } \\
(3,21-4,93)\end{array}$ & $\begin{array}{l}\text { patients intubated on-site had } \\
\text { 4-fold risk of dying; } \\
\text { patients intubated by using } \\
\text { medication showed better } \\
\text { survival. }\end{array}$ \\
\hline 45 & $\begin{array}{l}\text { Cameron et al. } \\
2005 \\
\text { Australia }\end{array}$ & $\begin{array}{l}\text { before-after-design } \\
\text { all prehospital patients } \\
\text { ALS-PHYSICIAN } n=211 \\
\text { ALS-PARAMEDIC BLS } n=163\end{array}$ & $\begin{array}{l}\text { ALS-PHYSICIAN, ALS- } \\
\text { PARAMEDIC } \\
\text { no BLS-group. } \\
\text { ALS-PHYSICIAN } \\
\text { helicopter } \\
\text { ALS-PARAMEDIC } \\
\text { helicopter }\end{array}$ & $\begin{array}{l}30 \text { days mortality } \\
\text { ALS-PHYSICIAN 2,8\% } \\
\text { ALS-PARAMEDIC 2,5\%, } \\
\text { NS }\end{array}$ & $\begin{array}{l}\text { no difference bewtween ALS- } \\
\text { PHYSICIAN and ALS-PARAMEDIC } \\
\text {-groups }\end{array}$ \\
\hline 46 & $\begin{array}{l}\text { Mellado Vergel } \\
\text { et al. } 2005 \\
\text { Spain }\end{array}$ & $\begin{array}{l}\text { retrospective } \\
\text { cardiac infarct, } \mathrm{PHT} \\
\mathrm{PHT} \mathrm{n}=152(\mathrm{ALS}) \text {, hospital } \\
\text { trombolysis (BLS) } \mathrm{n}=829\end{array}$ & $\begin{array}{l}\text { ALS-PARAMEDIC } \\
\text { BLS-EMT } \\
\text { ALS GA, BLS GA }\end{array}$ & $\begin{array}{l}30 \text { days mortality } \\
\text { ALS 5,9\%, BLS 26,6\% ( } p= \\
0,066)\end{array}$ & $\begin{array}{l}\text { ALS }(\mathrm{PHT}) \text { showed a trend to } \\
\text { lower mortality }\end{array}$ \\
\hline 47 & $\begin{array}{l}\text { Di Bartolomeo } \\
\text { et al. } 2005 \\
\text { Italy }\end{array}$ & $\begin{array}{l}\text { prospective } \\
\text { traumatic cardiac arrest } \\
\text { (blunt trauma) } \\
\text { ALS } n=56, \text { BLS } n=73\end{array}$ & $\begin{array}{l}\text { ALS-PHYSICIAN } \\
\text { BLS-EMT+BLS-nurse } \\
\text { ALS helicopter, BLS GA }\end{array}$ & $\begin{array}{l}\text { ALS } 96,5 \% \\
\text { only two patients } \\
\text { survived } \\
\text { BLS } 100 \% \text {, NS }\end{array}$ & $\begin{array}{l}\text { no difference between ALS and } \\
\text { BLS groups. } \\
\text { prognosis still very poor }\end{array}$ \\
\hline 48 & $\begin{array}{l}\text { Davis et al. } \\
2005 \\
\text { California, U.S. } \\
\text { A. }\end{array}$ & $\begin{array}{l}\text { retrospective epidemiological } \\
\text { study } \\
\text { brain injury } \\
\text { ALS-helicopter } n=3017 \\
\text { ALS- GA } n=7295\end{array}$ & $\begin{array}{l}\text { Helicopter manned by } \\
\text { paramedic, physician or } \\
\text { nurse, } \\
\text { ambulances manned by } \\
\text { paramedics } \\
\text { ALS helicopter, ALS GA }\end{array}$ & $\begin{array}{l}\text { mortality: ALS helicopter } \\
25,2 \% \\
\text { ALS ground ambulance } \\
25,3 \% \\
\text { Adjusted OR } 1,90(1,60- \\
2,25) \\
\text { mortality of patients } \\
\text { intubated on site: ALS- } \\
\text { helicopter } 42,5 \% \\
\text { ALS-GA } 43,1 \% \text {, OR } 1,42 \\
(1,13-1,78)\end{array}$ & $\begin{array}{l}\text { ALS + helicopter + intubation } \\
\text { on site better than ALS +GA + } \\
\text { intubation in hospital }\end{array}$ \\
\hline 49 & $\begin{array}{l}\text { Björklund et al. } \\
\text { Sweden, } 2006\end{array}$ & $\begin{array}{l}\text { prospective } \\
\text { prehospital trombolysis } \\
\text { ALS } n=1690 \\
\text { BLS } n=3685 \\
\text { comparison between PHT } \\
\text { entered in ambulance and } \\
\text { thrombolysis in hospital }\end{array}$ & $\begin{array}{l}\text { ALS-PARAMEDIC } \\
\text { BLS-EMT, GA in both } \\
\text { groups }\end{array}$ & $\begin{array}{l}\text { mortality: ALS 5,4\%, BLS } \\
8,3 \\
p<0,001 \text {. ALS 0,71 }(0,55- \\
0,92) \text { (1 year mortality); } \\
\text { ALS 0,79 }(0,61-1,03) 30 \\
\text { day mortality }\end{array}$ & ALS showed lower mortality \\
\hline 50 & $\begin{array}{l}\text { Sukumaran et } \\
\text { al. } 2006 \\
\text { Scotland }\end{array}$ & $\begin{array}{l}\text { prospective TRISS } \\
\text { all trauma patients } \\
\text { ALS } n=12339 \\
\text { BLS } n=9078\end{array}$ & $\begin{array}{l}\text { ALS-PARAMEDIC } \\
\text { BLS-EMT } \\
\text { ALS GA, BLS GA }\end{array}$ & $\begin{array}{l}\text { mortality: ALS 5,3\%, BLS } \\
4,5 \% \\
p=0,07 ; \text { after } \\
\text { adjustment no difference } \\
\text { between groups }\end{array}$ & $\begin{array}{l}\text { no difference between ALS and } \\
\text { BLS groups }\end{array}$ \\
\hline
\end{tabular}


Table 2 Summary of findings in the articles presenting effectiveness of advanced vs. basic life support. (Continued)

\begin{tabular}{|c|c|c|c|c|c|}
\hline 51 & $\begin{array}{l}\text { lirola et al. } \\
2006 \\
\text { Finland }\end{array}$ & $\begin{array}{l}\text { retrospective before-after } \\
\text { multiple trauma } \\
\text { ALS } n=81, \text { BLS } n=77\end{array}$ & $\begin{array}{l}\text { ALS-PHYSICIAN, BLS- } \\
\text { EMT } \\
\text { ALS helicopter (60\%) or } \\
\text { GA (39\%) } \\
\text { BLS GA }\end{array}$ & $\begin{array}{l}\text { mortality: ALS 31\%, BLS } \\
18 \% \\
\text { p = 0,065; TRISS: material } \\
\text { does not fit with MTOS- } \\
\text { material } \\
\text { QoL: no difference } \\
\text { between groups }\end{array}$ & $\begin{array}{l}\text { no difference between ALS and } \\
\text { BLS groups, } \\
\text { trend to bigger mortality in ALS- } \\
\text { group }(p=0,065)\end{array}$ \\
\hline 52 & $\begin{array}{l}\text { Klemen and } \\
\text { Grmec } 2006 \\
\text { Slovenia }\end{array}$ & $\begin{array}{l}\text { prospective, historical controls } \\
\text { multiple trauma, isolated head } \\
\text { injury } \\
\text { ALS } n=64, \text { BLS } n=60\end{array}$ & $\begin{array}{l}\text { ALS-PHYSICIAN, ALS- } \\
\text { EMT } \\
\text { ALS GA, BLS GA }\end{array}$ & $\begin{array}{l}\text { mortality ALS 40\%, BLS } \\
42 \% \text { (NS). GOS level } 4-5 \\
\text { achieved: ALS 53\%, BLS } \\
33 \%, p<0,01\end{array}$ & $\begin{array}{l}\text { no difference in mortality } \\
\text { in ALS better QoL }\end{array}$ \\
\hline 53 & $\begin{array}{l}\text { Stiell et al. } \\
2007 \\
\text { Canada }\end{array}$ & $\begin{array}{l}\text { prospective before-after } \\
\text { dyspnoea, ALS } n=4218, \text { BLS n } \\
=3920\end{array}$ & $\begin{array}{l}\text { BLS-EMT, ALS- } \\
\text { PARAMEDIC } \\
\text { ALS GA, BLS GA }\end{array}$ & $\begin{array}{l}\text { ALS } 11,3 \% \\
\text { BLS } 13,1 \%(p=0,01)\end{array}$ & lower mortality in ALS \\
\hline 54 & $\begin{array}{l}\text { Woodall et al. } \\
2007 \\
\text { Australia }\end{array}$ & $\begin{array}{l}\text { retrospective } \\
\text { cardiac arrest } \\
\text { ALS } n=1687 \\
\text { BLS } n=1288\end{array}$ & $\begin{array}{l}\text { ALS-PARAMEDIC } \\
\text { BLS-EMT } \\
\text { ALS GA, BLS GA }\end{array}$ & $\begin{array}{l}\text { mortality: ALS 93,3\%, BLS } \\
95,3 \% \text {; probablility for } \\
\text { survival in all patients } \\
\text { BLS }=1, \text { ALS }=1,43 \\
(1,02-1,99)\end{array}$ & lower mortality ALS \\
\hline 55 & $\begin{array}{l}\text { Ma et al. } 2007 \\
\text { Taiwan }\end{array}$ & $\begin{array}{l}\text { prospective } \\
\text { cardiac arrest } \\
\text { ALS } n=386 \\
\text { BLS } n=1037\end{array}$ & $\begin{array}{l}\text { ALS-PARAMEDIC, BLS- } \\
\text { EMT } \\
\text { ALS GA, BLS GA }\end{array}$ & $\begin{array}{l}\text { mortality ALS 93\%, BLS } \\
\text { 95\% (NS); survival in ALS } \\
\text { adjusted OR 1,41 (0,85- } \\
2,32)\end{array}$ & no difference between groups \\
\hline 56 & $\begin{array}{l}\text { Seamon et al. } \\
2007 \\
\text { Pennsylvania, } \\
\text { U.S.A. }\end{array}$ & $\begin{array}{l}\text { retrospective } \\
\text { patients going to immediate } \\
\text { thoracotomy comparison } \\
\text { between ALS or BLS ( } n=88) \\
\text { and private transport by } \\
\text { laymen } n=92\end{array}$ & $\begin{array}{l}\text { ALS-PARAMEDIC ori } \\
\text { BLS-EMT, } \\
\text { compared to } \\
\text { transportation by } \\
\text { laymen. }\end{array}$ & $\begin{array}{l}\text { mortality ALS,BLS } 92 \% \\
\text { private transport } 82,6 \% \\
\text { in multivariate analysis } \\
\text { prehospital procedures } \\
\text { were an independent } \\
\text { predictor of mortality }\end{array}$ & $\begin{array}{l}\text { better survival in persons } \\
\text { transported by laymen }\end{array}$ \\
\hline 57 & $\begin{array}{l}\text { Stiell et al. } \\
2008 \\
\text { Canada }\end{array}$ & $\begin{array}{l}\text { Before-after -design. } \\
92 \% \text { blunt trauma, (ISS > 12), } \\
\text { age } \geq 16 \text { years } \\
\text { ALS } n=1494 \\
\text { BLS } n=1373 \\
\text { Only } 72 \% \text { of the patients were } \\
\text { transferred directly to the } \\
\text { trauma centers from the scene. }\end{array}$ & $\begin{array}{l}\text { ALS-PARAMEDIC, GA. } \\
\text { BLS-PARAMEDIC, GA. } \\
\text { Endotracheal intubation } \\
\text { (7\%), iv fluid (12\%) and } \\
\text { drug administration } \\
\text { during the latter period. }\end{array}$ & $\begin{array}{l}\text { Mortality ALS 18,9\%, } \\
\text { BLS 18,2\% }(p=0,65) \\
\text { In patients with GCS < } \\
\text { mortality ALS 49,1\%, } \\
\text { BLS } 40,0 \%(p=0,02)\end{array}$ & $\begin{array}{l}\text { Implemantation of ALS did not } \\
\text { decrease mortality or morbidity. } \\
\text { In more severely injured } \\
\text { patients (GCS }<9 \text { ), mortality was } \\
\text { lower in the BLS group. }\end{array}$ \\
\hline
\end{tabular}

Abbreviations: ALS = advanced life support, BLS = basic life support, EMT = emergency medical technician, LOS = length of stay (in hospital), ISS $=$ Injury severity scale/score, TRISS = Trauma Score - Injury Severity Score, HEMS = Helicopter emergency medical service, GA = ground ambulance. PHT = prehospital throbolysis, $\mathrm{OR}=$ odds ratio, $\mathrm{RR}=$ risk ratio, ALS-N = advanced life support - nurse, ISS = injury severity score. QoL = quality of life.

\section{Multiple blunt injury}

Eight studies concerned patients with multiple blunt injuries [33,35,37,41,47,51,52,57]. No clear difference between ALS and BLS was found $[47,51,52,57]$. Two studies showed better results for BLS [33,35]. Results were difficult to estimate, because the comparison between ALS and BLS was confounded by transportation (helicopter or GA) and treatment level of the hospital [41].

\section{Respiratory distress}

The effect of prehospital treatment for patients having respiratory distress was studied in two papers [32,53], both of them favouring ALS.

\section{Other diseases}

One study was focused on epileptic patients [14] and one study on all unconscious patients (epilepsy, hypoglycaemia or stroke) [15]. In epileptic emergencies, the results favour ALS [14], and in the other study no difference was detected [15]. Hardly any research exists on several patient groups needing emergency care (e.g. stroke, intoxication, drowning,).

\section{Discussion}

The most remarkable limitation in this study is that definition of ALS and BLS is changing in time and place. This main problem is followed by several other problems:

1. Both ALS and BLS have developed and some methods used formerly in ALS may later be included into BLS.

2. Different studies use also different definitions of ALS and BLS.

3. The inclusion and exclusion criteria of this study have been set according to one definition. If the definition is changed, the set of articles may also be different. 
4. The level of credibility of separate articles is always subjective, especially in articles with borderline credibility.

In studies concerning unselected patient groups, the evidence does not support ALS. Similarly, in studies of all injuries, ALS has not been found to be superior compared to BLS. ALS treatment by a paramedic can even be harmful compared to BLS. When the patient material has concentrated to more serious cases, blunt head injuries or multiple injuries, some studies have demonstrated a beneficial effect of ALS. However, not all studies confirm this. ALS seems to be most beneficial when having an experienced physician in the staff. The critical limit of the experience of the staff can be defined as the ability to perform an intubation by using hypnotics and muscle relaxants. If this limit has not been reached, an ALS activity can be harmful.

We did not find evidence supporting ALS in regard to cardiac arrest if use of a defibrillator is included into BLS. In cardiac arrest, early cardiopulmonary resuscitation (CPR) and defibrillation are essential for a patient's survival. For instance in Finland, even lay rescuers are trained to use an automated external defibrillator and start CPR. No evidence has been found on the effectiveness of other activities in treating sudden cardiac arrest when the end point of the studies has been secondary survival (at discharge from hospital). The most promising intervention - prehospitally initiated therapeutic hypothermia - still needs more validation.

Prehospital thrombolysis seems to be superior to thrombolysis given in a hospital, but it should be remembered that prehospital thrombolysis is only one link in the treatment chain. We have not assessed the effect of other treatments for acute myocardial infarction.

We interpret the contradictory findings between studies as due mainly to the multitude of definitions for ALS and BLS, differences in treatment populations and interventions, the high risk of bias in the original studies, and lack of statistical power: cases where ALS is beneficial may be too rare to be identified by statistical methods in an unselected material. There is a clear need for international definitions of ALS and BLS, appropriate documentation of populations and performed interventions in trials, and more high quality studies taking into account different patient groups. Among defined patients, e.g. those having brain injuries, the benefit of ALS can be found if there are enough eligible patients. The infrequency of potential cases for ALS indicates that an ALS emergency system requires a large enough catchment area.

Only a very few studies analyzing prehospital care of trauma described the severity of trauma and the interventions performed. The control groups were usually not comparable to the index group: the ALS groups tend to consist of more severely injured patients. Additionally, common confounding factors include different transportation means (helicopter vs. ground ambulances) and the different levels of the admitting trauma hospitals. In many studies patients with only very mild injuries or, conversely, patients with little chance of survival (i.e. traumatic cardiac arrest or gunshot wounds to head) have been included.

The majority of studies favour the use of a helicopter, but the results were contradictory. In many studies, the effectiveness of an operation model (ALS vs. BLS) was confounded by not being able to take into account the vehicle used (helicopter vs. ambulance).

The effectiveness of a helicopter may warrant that it is used for all patient groups, and especially for patients where prehospital treatment is known to be effective (e. g. myocardial infarction). Among trauma patients, the best results are probably achieved when the severity of the trauma can be classified as being moderate or serious but not indicating a poor prognosis. A helicopter service requires a sufficiently large catchment population.

The distance from the site to the hospital mediates the effectiveness of ALS. For short distances (urban and semi-urban areas), there is no evidence favouring ALS in the case of an injury. According to Nicholl et all [58] a $10-\mathrm{km}$ increase in distance from a hospital is associated with a $1 \%$ absolute increase in mortality. For longer distance, ALS operating with a helicopter seems to be effective, but still there is a contradiction in terms of cost-effectiveness: longer distances are associated with a more sparse population. In a British study concerning trauma patients only [7], the population base was estimated to be 3 million. In Finland, injuries make only about $20 \%$ of all cases in a typical Finnish Helicopter Emergency Medical Service. Thus the estimation by Nicholl is in line with a Finnish practical experience that a population of 0.5 million to be reached in 30 minutes may be satisfactory for a helicopter-ALS -service.

While the concepts "scoop and run" or "stay and play" are often present in the literature, they reflect the tactics of emergency services, and should not be seen to correlate directly with ALS or BLS. An ALS-level emergency unit employs rapid assessment and scoop and run -tactics when a definitive treatment outside the hospital is not possible. A penetrating injury with bleeding is an example of a situation where immediate surgical treatment is imperative.

When a diagnosis is feasible and definitive, an effective treatment can be started prehospital by using stay and play -tactics, for example, prehospital thrombolysis in a 
myocardial infarct and early defibrillation in a cardiac arrest.

The tactic of choice is determined by the nature of the emergency, the available services, and the possibilities for starting the treatment in the hospital. All hospitals can not give all treatments at all times of the day. Treatment delays are caused not only by distances but also by the care level of the hospital.

The right to emergency health care services in many countries (including Finland) is granted to citizens by a legal constitution. Equal access to effective treatment is also one of the fundamental principles of health care in many countries. How to organize an emergency service is affected by medical knowledge but also by general opinion, people's sense of safety and earlier structures of services. It may be reasonable to organize ALS-level emergency services even when there is uncertainty about the cost-effectiveness of such services. An ALS system operating with a helicopter can also bring services to sparsely populated areas.

\section{Conclusions}

The overall quality of the analyzed studies was poor. Among 1333 studies, only one randomised controlled trial was found. Thus, no conclusions on the effectiveness of advanced prehospital care in unselected patient cohorts can be drawn.

In the prehospital care of sudden cardiac arrest early defibrillation and cardiopulmonary resuscitation are still essential; further ALS interventions have not been able to demonstrate increased survival. Prehospitally initiated hypothermia is a promising treatment but is not as yet an evidence-based intervention.

Prehospitally initiated thrombolysis of myocardial infarction improves survival when compared with inhospital initiation of thrombolytic treatment.

There is evidence that ALS is beneficial in epileptic patients and among those with respiratory distress.

Due to multiple methodological problems found in trauma studies, the comparison of ALS and BLS prehospital care is difficult and, in unselected trauma cohorts, impossible. It seems obvious that in urban settings and in patients with penetrating injuries, ALS does not improve survival. In some patients, for instance, patients with severe head injuries, ALS provided by paramedics and intubation without anaesthesia can even be harmful due to uncontrolled intracranial pressure. If the prehospital care is provided by an experienced physician and by a HEMS organisation (Helicopter Emergency Medical Service), ALS interventions may be beneficial for patients with multiple blunt injuries.

In many patient groups such as patients with cerebrovascular problems, intoxication, drowning etc. there is very little research available on the effectiveness of ALS and BLS levels of prehospital care and thus no conclusions can be made.

The need for high quality controlled clinical studies is obvious. Besides that, the development of prehospital care requires uniform and full documentation and follow-up of patients as well as register studies based on real-life data.

\section{Acknowledgements}

The authors thank Riitta Grahn and Jaana Isojärvi from the library of The National Institute for Health and Welfare (former STAKES) for completing searches. Professor Marjukka Mäkelä has given important advice in planning the study.

\section{Author details}

${ }^{1}$ University of Eastern Finland, Department of Public Health and Clinical Nutrition, P.O. Box 1627, 70211 Kuopio, Finland. 'Kuopio University Hospital/ Primary Health Care, P.O. Box 1777, 70211 Kuopio, Finland. ${ }^{3}$ University of Turku and Turku University Hospital, Department of Anaesthesiology, Intensive Care, Emergency Care and Pain Medicine, P.O. Box 52, 20521 Turku, Finland. ${ }^{4}$ Töölö Hospital, Helsinki University Central Hospital, Department of Anesthesiology and Intensive Care Medicine, P.O. Box 266, 00029 HUS, Finland. ${ }^{5}$ Finnish Medical Association, P.O.Box 49, 00501 Helsinki, Finland. ${ }^{6}$ Centre for Health and Social Economics, Insitute for Health and Welfare, Mannerheimintie 166, 00270 Helsinki, Finland.

\section{Authors' contributions}

$\mathrm{OPR}, \mathrm{TI}, \mathrm{JR}, \mathrm{HP}$ and $\mathrm{AM}$ all have been participating in the study design and planning the search strategy. OPR, TI, JR and AM assessed the articles and made the data extraction. The manuscript was completed by OPR, TI and JR, assisted by AM. All authors have read and approved the final manuscript.

\section{Competing interests}

$J R$ is a doctor-in-charge of MediHeli helicopter emergency medical service, Helsinki, Finland. TI is a clinician of MediHeli 02, Turku, Finland. Other authors declare that they have no competing interests.

Received: 11 June 2010 Accepted: 23 November 2010 Published: 23 November 2010

\section{References}

1. Liberman M, Roudsari BS: Prehospital trauma care: what do we really know? Curr Opin Crit Care 2007, 13:691-6.

2. Isenberg $D L$, Bissell $R$ : Does advanced life support provide benefits to patients?: A literature review. Prehospital Disaster Med 2005, 20:265-70.

3. Gold CR: Prehospital advanced life support vs "scoop and run" in trauma management. Ann Emerg Med 1987, 16:797-801.

4. Ryynänen O-P, lirola T, Reitala J, Pälve H, Malmivaara A: Ensihoidon vaikuttavuus. Järjestelmällinen kirjallisuuskatsaus. (Effectiveness of prehospital care. A systematic review) Finohta report 32/2008. Finnish with Swedish and English abstract [http://finohta.stakes.fi/EN/index.htm].

5. Liberman M, Mulder D, Sampalis JS: Advanced or basic life support for trauma: meta-analysis and critical review of the literature. J Trauma 2000, 49:584-599.

6. Sethi D, Kwan I, Kelly AM, Roberts I, Bunn F: Advanced trauma life support training for ambulance crews. Cochrane Database Syst Rev 2001, , 2: CD003109.

7. Nicholl J, Turner J, Stevens K, O'Keeffe C, Cross L, Goodacre S, Snooks H: A review of the costs and benefits of helicopter emergency ambulance services in England and Wales. Final report to the Department of Health. University of Sheffield, Medical Care Research Unit, Sheffield; 2003 [http:// www.shef.ac.uk/uni/academic/R-Z/scharr/mcru/reports/HEASrev.pdf].

8. Koskinen $\mathrm{H}$ : Lääkintähelikopterin vaikuttavuus ja kustannusvaikuttavuus. (Effectiveness and cost-effectiveness of a helicopter emergency service, in Finnish). Thesis, University of Kuopio, Kuopio 2005.

9. Thomas SH, Cheema F, Wedel SK, Thomson D: Trauma helicopter emergency medical services transport: annotated review of selected outcomes-related literature. Prehosp Emerg Care 2002, 6:359-71. 
10. Thomas SH: Helicopter emergency medical services transport outcomes literature: annotated review of articles published 2000-2003. Prehosp Emerg Care 2004, 8:322-33.

11. Thomas SH: Helicopter EMS transport outcomes literature: annotated review of articles published 2004-2006. Prehosp Emerg Care 2007, 11:477-88.

12. Shuster M, Keller J, Shannon H: Effects of prehospital care on outcome in patients with cardiac illness. Ann Emerg Med 1995, 26:138-45.

13. Boissel JP: The European Myocardial Infarction Project: an assessment of pre-hospital thrombolysis. Int J Cardiol 1995, 49(Suppl):S29-37.

14. Alldredge $B K$, Wall $D B$, Ferriero DM: Effect of prehospital treatment on the outcome of status epilepticus in children. Pediatr Neurol 1995, , 12: 213-6.

15. Adams J, Aldag G, Wolford R: Does the level of prehospital care influence the outcome of patients with altered levels of consciousness? Prehosp Disaster Med 1996, 11:101-4.

16. Demetriades D, Chan L, Cornwell E, et al: Paramedic vs private transportation of trauma patients. Arch Surg 1996, 131:133-138.

17. Silfvast $T$, Ekstrand $A$ : The effect of experience of on-site physicians on survival from prehospital cardiac arrest. Resuscitation 1996, 31:101-5.

18. Nguyen-Van-Tam JS, Dove AF, Bradley MP, Pearson JC, Durston P, Madeley R: Effectiveness of ambulance paramedics versus ambulance technicians in managing out of hospital cardiac arrest. J Accid Emerg Med 1997, 14:142-8.

19. Rainer TH, Houlihan KP, Robertson CE, Beard D, Henry JM, Gordon MW: An evaluation of paramedic activities in prehospital trauma care. Injury 1997, 28(9-10):623-7.

20. Rainer TH, Marshall R, Cusack S: Paramedics, technicians, and survival from out of hospital cardiac arrest. J Accid Emerg Med 1997, 14:278-82.

21. Suominen P, Baillie C, Kivioja A, Korpela R, Rintala R, Silfvast T, Olkkola KT: Prehospital care and survival of pediatric patients with blunt trauma. $J$ Pediatr Surg 1998, 33:1388-92.

22. Nicholl JP, Brazier JE, Snooks HA: Effects of London helicopter emergency medical service on survival after trauma. BMJ 1995, 311:217-22.

23. Eisen JS, Dubinsky I: Advanced life support vs basic life support field care: an outcome study. Acad Emerg Med 1998, 5:592-8.

24. Abbott D, Brauer K, Hutton K, Rosen P: Aggressive out-of-hospital treatment regimen for severe closed head injury in patients undergoing air medical transport. Air Med J 1998, , 17: 94-100.

25. Owen JL, Phillips RT, Conaway C, Mullarkey D: One year's trauma mortality experience at Brooke Army Medical Center: is aeromedical transportation of patients necessary? Military Medicine 1999, 164:361-365.

26. Mitchell RG, Guly UM, Rainer TH, Robertson CE: Paramedic activities, drug administration and survival from out of hospital cardiac arrest. Resuscitation 2000, 43:95-100

27. Eckstein M, Chan L, Schneir A, Palmer R: Effect of prehospital advanced life support on outcomes of major trauma patients. J Trauma 2000, 48:643-648.

28. Pitetti R, Glustein JZ, Bhende MS: Prehospital care and outcome of pediatric out-of-hospital cardiac arrest. Prehosp Emerg Care 2002, 6:283-90.

29. Garner AA: The role of physician staffing of helicopter emergency medical services in prehospital trauma response. Emerg Med Australas 2004, 16:318-23.

30. Di Bartolomeo S, Sanson G, Nardi G, Scian F, Michelutto V, Lattuada L: Effects of 2 patterns of prehospital care on the outcome of patients with severe head injury. Arch Surg 2001, 136:1293-300.

31. Kurola J, et al: Paramedic helicopter emergency service in rural Finland do benefits justify the cost? Acta Anaesthesiol Scandinavia 2002, 46:779-784.

32. Bjerre SK, Hansen TM, Melchiorsen H, Christensen EF: Prehospital treatment of patients with acute exacerbation of chronic pulmonary disease. Before and after introduction of a mobile emergency unit. Ugeskr Laeger 2002, 164:1349-52.

33. Thomas SH, et al: Helicopter transport and blunt trauma mortality: a multicenter trial. J Trauma 2002, 52:136-145.

34. Lossius HM, Søreide E, Hotvedt R, Hapnes SA, Eielsen OV, Førde OH, Steen PA: Prehospital advanced life support provided by specially trained physicians: is there a benefit in terms of life years gained? Acto Anaesthesiol Scand 2002, 46:771-8.

35. Lee A, Garner A, Fearnside M, Harrison K: Level of prehospital care and risk of mortality in patients with and without severe blunt head injury. Injury 2003, 34:815-9.
36. Christenszen EF, Melchiorsen H, Kilsmark J, Foldspang A, Søgaard J: Anesthesiologists in prehospital care make a difference to certain groups of patients. Acta Anaesthesiol Scand 2003, 47:146-52.

37. Osterwalder Jj: Mortality of blunt polytrauma: a comparison between emergency physicians and emergency medical technicians-prospective cohort study at a level I hospital in eastern Switzerland. J Trauma 2003, 55:355-61

38. Bochicchio GV, llahi O, Joshi M, et al: Endotracheal intubation in the field does not improve outcome in trauma patients who present without an acutely lethal traumatic brain injury. I Trauma 2003, 54:307-311.

39. Liberman M, Mulder D, Lavoie A, et al: Multicentre Canadian study of prehospital trauma care. Ann Surg 2003, 237:153-160.

40. Danchin N, Blanchard D, Steg PG, Sauval P, Hanania G, Goldstein P, Cambou JP, Guéret P, Vaur L, Boutalbi Y, Genès N, Lablanche JM, USIC 2000 Investigators: Impact of prehospital thrombolysis for acute myocardial infarction on 1-year outcome: results from the French Nationwide USIC 2000 Registry. Circulation 2004, 110:1909-15.

41. Wang HE, Peitzman AB, Cassidy LD, Adelson PD, Yealy DM: Out-of-hospital endotracheal intubation and outcome after traumatic brain injury. Ann Emerg Med 2004, 44:439-50

42. Biewener A, Aschenbrenner U, Rammelt S, Grass R, Zwipp H: Impact of helicopter transport and hospital level on mortality of polytrauma patients. J Trauma 2004, 56:94-8.

43. Stiell IG, Wells GA, Field B, Spaite DW, Nesbitt LP, De Maio VJ, Nichol G, Cousineau D, Blackburn J, Munkley D, Luinstra-Toohey L, Campeau T, Dagnone E, Lyver M, Ontario Prehospital Advanced Life Support Study Group: Advanced cardiac life support in out-of-hospital cardiac arrest. N Engl J Med 2004, 351:647-56.

44. Frankema SP, Ringburg AN, Steyerberg EW, Edwards MJ, Schipper IB, van Vugt AB: Beneficial effect of helicopter emergency medical services on survival of severely injured patients. Br J Surg 2004, 91:1520-6.

45. Cameron S, Pereira P, Mulcahy R, Seymour J: Helicopter primary retrieval: tasking who should do it? Emerg Med Australas 2005, 17:387-91.

46. Mellado Vergel FJ, Rosell Ortiz F, Ruiz Bailén M, Grupo PEFEX: Out-ofhospital treatment of acute myocardial infarction in Andalusia, Spain. Rev Esp Cardiol 2005, 58:1287-93.

47. Di Bartolomeo S, Sanson G, Nardi G, Michelutto V, Scian F: HEMS vs. Ground-BLS care in traumatic cardiac arrest. Prehosp Emerg Care 2005, 9:79-84.

48. Davis DP, Peay J, Serrano JA, Buono C, Vilke GM, Sise MJ, Kennedy F, Eastman AB, Velky T, Hoyt DB: The impact of aeromedical response to patients with moderate to severe traumatic brain injury. Ann Emerg Med 2005, 46:115-22.

49. Björklund E, Stenestrand U, Lindbäck J, Svensson L, Wallentin L, Lindahl B: Pre-hospital thrombolysis delivered by paramedics is associated with reduced time delay and mortality in ambulance-transported real-life patients with ST-elevation myocardial infarction. Eur Heart J 2006, 27:1146-52.

50. Sukumaran S, Henry JM, Beard D, Lawrenson R, Gordon MW, O'Donnell JJ, Gray AJ: Prehospital trauma management: a national study of paramedic activities. Emerg Med J 2005, 22:60-3.

51. lirola TT, Laaksonen MI, Vahlberg TJ, et al: Effect of physician-staffed helicopter emergency medical service on blunt trauma patient survival and prehospital care. Eur J Emerg Med 2006, 13:335-339.

52. Klemen P, Grmec S: Effect of pre-hospital advanced life support with rapid sequence intubation on outcome of severe traumatic brain injury. Acta Anaesthesiol Scand 2006, 50:1250-4.

53. Stiell IG, Spaite DW, Field B, Nesbitt LP, Munkley D, Maloney J, Dreyer J, Toohey LL, Campeau T, Dagnone E, Lyver M, Wells GA, OPALS Study Group: Advanced life support for out-of-hospital respiratory distress. N Engl J Med 2007, 356:2156-64.

54. Woodall J, McCarthy M, Johnston T, Tippett V, Bonham R: Impact of advanced cardiac life support-skilled paramedics on survival from outof-hospital cardiac arrest in a statewide emergency medical service. Emerg Med J 2007, 24:134-8.

55. Ma MH, Chiang WC, Ko PC, Huang JC, Lin CH, Wang HC, Chang WT, Hwang $\mathrm{CH}$, Wang YC, Hsiung GH, Lee BC, Chen SC, Chen WJ, Lin FY: Outcomes from out-of-hospital cardiac arrest in Metropolitan Taipei: does an advanced life support service make a difference? Resuscitation 2007, 74:461-9. 
56. Seamon MJ, Fisher CA, Gaughan J, et al: Prehospital procedures before emergency department thoracotomy: 'scoop and run' saves lives. J Trauma 2007, 63:113-120

57. Stiell IG, Nesbitt LP, Pickett W, Munkley D, Spaite DW, Banek J, Field B, Luinstra-Toohey L, Maloney J, Dreyer J, Lyver M, Campeau T, Wells GA, the OPALS Study Group: The OPALS Major Trauma Study: impact of advanced life-support on survival and morbidity. CMAJ 2008, 178:1141-52

58. Nicholl J, West J, Goodacre S, Turner J: The relationship between distance to hospital and patient mortality in emergencies: an observational study. Emerg Med J 2007, , 24: 665-8.

doi:10.1186/1757-7241-18-62

Cite this article as: Ryynänen et al.: Is advanced life support better than basic life support in prehospital care? A systematic review. Scandinavian Journal of Trauma, Resuscitation and Emergency Medicine 2010 18:62.

Submit your next manuscript to BioMed Central and take full advantage of:

- Convenient online submission

- Thorough peer review

- No space constraints or color figure charges

- Immediate publication on acceptance

- Inclusion in PubMed, CAS, Scopus and Google Scholar

- Research which is freely available for redistribution

Submit your manuscript at www.biomedcentral.com/submit
C Biomed Central 